\title{
Embedding HCI in Developing Countries: Localizing Content, Institutionalizing Education and Practice
}

\author{
Andy Smith ${ }^{1}$, Anirudha Joshi ${ }^{2}$, Zhengjie Liu ${ }^{3}$, Liam Bannon ${ }^{4}$, \\ Jan Gulliksen ${ }^{5}$, and Cecilia Baranauskas ${ }^{6}$ \\ ${ }^{1}$ Thames Valley University United Kingdom \\ andy.smithatvu.ac.uk \\ ${ }^{2}$ Industrial Design Centre, IIT Bombay, Mumbai, India \\ anirudha@itb.ac.in \\ ${ }^{3}$ Sino-European Usability Center, Dalian Maritime University \\ liuzhj@almu.edu.cn \\ ${ }^{4}$ Interaction Design Centre, University of Limerick, Ireland \\ liam.bannon@ul. ie \\ ${ }^{5}$ Uppsala University, Sweden \\ jan.gulliksen@it.uu.se \\ ${ }^{6}$ Institute of Computing, Unicamp, Brazil \\ cecilia@ic.unicamp.br
}

\begin{abstract}
This SIG will facilitate a debate concerning how best to support the development of indigenous HCI in developing countries, both as part of education and training systems and within industrial practice.
\end{abstract}

\section{Background}

There are signs of a new world economic and political order, where countries like Brazil, China, India and South Africa are emerging key players. There is also a developing awareness of their increased economic and political "muscle", especially as they begin to develop bilateral and multilateral agreements among themselves, increasing their strategic importance on the world stage. Some of these countries have rapidly developing IT industries supporting local (domestic) and / or global (offshoring) software development. In contrast to previous approaches, some are beginning to appreciate that a human-centred design approach to the development and use of ICT is critical both to maximize trade in local and global markets, and also in the socially-responsible development of their individual information societies.

In this SIG we will seek to explore how indigenous approaches to the humancentred ICT should shape the socially-responsible development of information societies in developing countries. The human-centred approach that we refer to here is of key importance in major segments of the ICT industry, in software applications for business and industry, in consumer markets, and in areas of health and defence. Successful growth of the ICT sectors in these countries, focusing on both internal and external markets, will be dependent on paying increased attention to human, social and cultural factors.

The number of usability professionals is growing significantly in those developing countries that have a significant ICT industry. Through a wide range of engagements 
with usability practitioners it is clear to the organisers that there is a considerable appetite to learn about Western HCI case studies in the expectation that these can be implemented locally. However there are two problems to overcome. Firstly a richer, more nuanced, understanding of HCI is necessary in order for the most effective tools or techniques to be successfully selected and implemented - this implies a much broader 'education in HCI' rather than just 'training in tools'. We need to be able to judge the appropriateness of particular tools for particular tasks, and this requires HCI education, not simply training in techniques. Secondly the cultural and organisational differences between countries mean that HCI tools and techniques that have been developed in Western countries may not be effective in developing countries. What is required is the localisation of methods to meet local requirements.

\section{Aims}

We seek to discuss the building of effective localised HCI in developing countries by addressing issues such as:

- The extent to which the discipline of HCI is changed in different countries;

- does software offshoring skew the tools and techniques adopted?

- is culture significant in changing processes and how do we identify these?

- How do we gain national / institutional support for HCI;

- so that HCI can feature in university curricular?

- and so that professional accreditation can be developed?

- How do we promote best practice in industry,

- both for global (offshoring) and local (domestic) systems development?

Note: The organisers of this SIG have liaised with those organising another SIG (Sharing Perspectives on Community-Centered Design and International Development). Whilst the two SIGs share a common background, the SIG described here focuses on localization of methods, capacity building, education and institutional support. The other SIG focuses in development, participation and values.

\section{Organisers}

Andy Smith has project managed two EC funded projects (Indo European Systems Usability Partnership - IESUP - and Sino European Systems Usability Network SESUN) supporting HCI and usability in India and China. Liam Bannon has acted as a partner in both the IESUP and SESUN projects and manages other research projects in global software development. Jan Gulliksen has acted as a partner in both IESUP and SESUN and is Chair of IFIP WG 13.2 Methodology for User Centred Systems Design. Anirudha Joshi is based at the Industrial Design Centre and Indian Institute for technology Bombay, India and has been involved in IESUP and many local Indian HCI projects. Zhengjie Liu is Head of the Sino European Usability Center in Dalian, China and is the Chinese partner is SESUN. Cecilia Baranauskas is Associate Professor at the Institute of Computing - Unicamp, Brazil. 